\title{
INDEPENDENCE THEORIES AND GENERALIZED ZERO-ONE LAWS
}

\author{
LAWRENCE NEFF STOUT ${ }^{1}$
}

\begin{abstract}
In this paper an abstract characterization of the properties of independent events is given with examples from topology, probability, and Baire structures. Using this notion of independence, proofs of the HewittSavage and Kolmogorov zero-one laws are given which include the probabilistic case and the topological cases considered by Oxtoby, Christensen, and K. P. S. and M. Bhaskara Rao.
\end{abstract}

In recent years several papers have appeared giving Baire Category analogues of the zero-one laws of probability ([1], [2], [3], [6], and [9] for the Kolmogorov law and [8] for Hewitt-Savage). In each case, while the results are strictly analogous, the proof is new. The object of this paper is to give an abstract setting for zero-one laws using the particularly simple proofs of the probabilistic case but including the topological zero-one laws as well. The key element in this approach is the notion of an independence theory.

Recall that in a probability space independence is completely determined once one knows which finite sets of events are independent. Independence is well behaved with respect to the operations of the $\sigma$-algebra. Independence of sequences of $\sigma$-algebras is determined by independence of sequences of events. This situation is generalized as follows:

Definition. Let $\mathrm{A}$ be a type of structure such that if $A$ is a set equipped with an $\mathrm{A}$ structure and $A^{\prime} \subseteq A$, then there is a smallest $\mathrm{A}$ substructure, denoted $\mathrm{A}\left(A^{\prime}\right)$, with $A^{\prime} \subseteq \mathrm{A}\left(A^{\prime}\right)$. We will call a set $A$ equipped with such a structure an A structure of events.

Observe that the usual algebraic structures (lattices, $\sigma$-algebras, etc.) satisfy this definition, as will many other kinds of structure not defined in terms of operations and equations (topologies for instance).

Definition. Let $I$ be a set of countable sequences of elements of $A$. A countable sequence of A substructures of $A,\left(\alpha_{i}\right)_{i \in N}$, is called $I$-independent if every sequence $\left(a_{i}\right)$ with $a_{i} \in \alpha_{i}$ is in $I$.

A set $I$ of countable sequences of elements of $A$ is called an independence theory on $A$ relative to A structures if

(1) $\left(a_{i}\right)_{i \in \Gamma} \in I \Leftrightarrow\left(a_{\sigma i}\right)_{i \in \Gamma} \in I$ for any permutation $\sigma$;

Received by the editors February 16, 1976 and, in revised form, November 8, 1976.

AMS (MOS) subject classifications (1970). Primary 54H05, 60F20, 60B99.

${ }^{1}$ Research conducted while the author was at McGill University supported by a grant from Québec Ministery of Education. 
(2) $\left(a_{i}\right)_{i \in \Gamma} \in I \Leftrightarrow \forall_{n \in N}\left(a_{i}\right)_{i \in \Gamma \cap \downarrow n} \in I$, where $\downarrow n$ is the set of all natural numbers less than $n$;

(3) $\left(\alpha_{i}\right)_{i \in N}$ is an $I$-independent sequence of A-substructures of $A$ and if $\left\{P_{i}\right\}_{i \in \Omega}$ is a partition of $N$, then $\left(\mathrm{A}\left(\cup_{j \in P_{i}} \alpha_{j}\right)\right)_{i \in \Omega}$ is $I$-independent;

(4) $\left(a_{i}\right)_{i \in \Gamma} \in I$, then $\left(A\left(a_{i}\right)\right)_{i \in \Gamma}$ is $I$-independent.

Note that for any A structure of events and any collection of finite sequences, there is a smallest independence theory for which the sequences given are independent.

EXAMPLES.

(1) Independent sequences of events in a probability space form an independence theory with respect to $\sigma$-algebras.

(2) We say that a subset $S$ of a product $\prod_{i \in \Gamma} X_{i}$ does not restrict the factors in $\Gamma^{\prime} \subseteq \Gamma$ if for any pair of points $x$ and $y$ differing only in coordinates in $\Gamma^{\prime}$ we have $x \in S$ if and only if $y \in S$. A map does not depend on the factors in $\Gamma^{\prime}$ if its value is not altered by changing coordinates in $\Gamma^{\prime}$.

If $(X, T)=\prod_{i \in \Gamma}\left(X_{i}, T_{i}\right)$ is a product topological space, then there is an independence theory relative to sub-complete lattice structures on $T$ obtained as follows: an ordered $n$-tuple $\left(U_{1}, \ldots, U_{n}\right)$ is in $I$ if $n=1$ or if $n=k+1$ and

(a) every subsequence with $k$ elements is in $I$, and

(b) there is a set $\left\{\Gamma_{i} \mid \Gamma_{i} \subseteq \Gamma, i=1, \ldots, n\right\}$ such that $U_{i}$ does not restrict the factors in $\Gamma_{i}$ and $\Gamma_{i} \cup\left(\bigcap_{j \neq i} \Gamma_{j}\right)=\Gamma$.

(3) For a function space with the compact open topology a similar theory may be obtained from the set of finite sequences of subbasic open sets such that the compact sets involved are pairwise disjoint.

(4) Let $X$ be a topological space with an independence theory $I$ on its topology. We construct an independence theory $I^{\prime}$ on the set of almost open sets (or $B P$ sets-sets $B$ such that there is an open set $U$ with $B \triangle U$ of first category) by letting $\left(B_{1}, \ldots, B_{n}\right) \in I^{\prime}$ if there is an $n$-tuple $\left(U_{1}, \ldots, U_{n}\right) \in I$ with $B_{i} \triangle U_{i}$ of first category. If $I$ is an independence theory with respect to sup-complete lattices, $I^{\prime}$ will be an independence theory with respect to lattices $L$ with a specified sublattice $L^{\prime}$ such that $L$ has arbitrary sups of collections with no more than countably many members not in $L^{\prime}$. If $I$ is a theory relative to sup-complete pseudo-complemented lattices, $I^{\prime}$ will be a theory with respect to $\sigma$-algebras.

(5) (Theory obtained by restriction.) Let $X$ be a lattice with an independence theory $I$ relative to lattice structures. Then for any $A \in X$ there is an independence theory $I_{A}$ relative to lattice structures on the set of elements of $X$ less than or equal to $A$ given by the set of all sequences $\left(B_{i}\right)_{i \in \Gamma}$ such that there is a sequence $\left(A_{i}\right)_{i \in \Gamma} \in I$ with $B_{i}=A_{i} \cap A$. If $X$ is a sublattice of some bigger lattice, $A$ need not be in $X$, but only in the bigger lattice.

In the proofs of the zero-one laws in probability, the conclusion that an event has probability zero or one is usually obtained by showing that it is independent of itself. $\left(P(A)=P(A \cap A)=P(A)^{2}\right.$ so $P(A)=0$ or 1.) This 
motivates the following definition:

Definition. Let $(A, I)$ be an independence theory, then $a \in A$ is zero-one if $(a, a) \in I$.

Examples:

(6) An open set in the restricting factors theory on a product topological space is zero-one if and only if it does not restrict any factors; hence, it must be empty or all of $X$.

(7) If $(X, T, I)$ is a topological space with an independence theory on its topology, then an almost open set $B$ is zero-one relative to the theory $I^{\prime}$ of Example (4) if there are open sets $U_{1}$ and $U_{2}$ differing from $B$ by sets of first category $F_{1}$ and $F_{2}$ such that $\left(U_{1}, U_{2}\right) \in I$. Restricting to $X-\left(F_{1} \cup F_{2}\right)$ makes the two opens equal and hence zero-one. For the theory of Example (2) this will tell us that $B$ is meager or residual.

Defintion. Let $\left(\alpha_{i}\right)_{i \in N}$ be a sequence of A-structures, then a tail event is a member of $\bigcap_{n \in N} \mathrm{~A}\left(\cup_{i=n}^{\infty} \alpha_{i}\right)$.

Theorem (Kolmogorov Zero-One LAW). If $\left(\alpha_{i}\right)_{i \in N}$ is I-independent and $a$ is a tail event for $\left(\alpha_{i}\right)_{i \in N}$, then a is zero-one.

Proof (as in Loève [5, p. 230]). Let $a$ be a tail event. By axiom (3), $\left(\alpha_{1}, \ldots, \alpha_{n}, A\left(\cup_{i>n} \alpha_{i}\right)\right)$ is $I$-independent so for any $a_{i} \in \alpha_{i}$ the sequence $\left(a_{1}, \ldots, a_{n}, a\right) \in I$. Thus by axioms (1) and (2), $\left(A(a), \alpha_{1}, \ldots\right)$ is $I$-independent. Thus $\left(A(a), A\left(\cup_{i \in N} \alpha_{i}\right)\right)$ is $I$-independent, so $(a, a) \in I$.

Corollary. Let $(X, T)=\Pi_{i \in N}\left(X_{i}, T_{i}\right)$ be a product topological space. An almost open set $B$ which does not restrict any finite set of factors must be either residual or meager.

Proof. Let $I$ be the restricting factors theory on $T$ (as in Example (2)); then the theory $I^{\prime}$ on the set of almost open sets (as in Example (4)) is an independence theory relative to B structures where a B structure is a lattice $L$ with a sublattice $L^{\prime}$ such that $L$ has all sups with only countably many elements not in $L^{\prime}$. Here $L$ is the lattice of almost open sets and $L^{\prime}$ is the lattice of open sets.

Consider the sequence $\left(\beta_{i}\right)_{i \in N}$ of B structures where $\beta_{i}$ is the set of almost open sets differing by a set of first category from one of the subbasic open sets $\pi_{i}^{-1} U$, where $\pi_{i}$ is the projection onto the $i$ th factor. This is an $I^{\prime}$-independent sequence of $B$ structures. We need only show $B$ is a tail event.

Observe that since $B$ does not restrict the first $n$ factors $\pi^{-1} \pi B=B$ where $\pi$ is the projection removing the first $n$ factors. Since $B$ is almost open it may be written as a sum $B^{\prime}+F$ where $B^{\prime}=\cap_{i \in N} U_{i}$ is a $G_{\delta}$ and $F$ is of first category (see [7, p. 20]). We wish to replace $U_{i}$ by an open set not restricting the first $n$ factors. Let $U_{i}^{\prime}=\pi^{-1} \pi\left(\cap_{i=1, \ldots, n} U_{i}\right)$. Then $\bigcap_{i \in N} U_{i}^{\prime} \supseteq \bigcap_{i \in N} U_{i}$ and it will suffice to show that $\bigcap_{i \in N} U_{i}^{\prime} \subseteq B$.

If $x \notin B$ then no $y$ differing from $x$ only in the first $n$ coordinates is in $B$. There are finitely many such $y$ 's so there is an $m$ such that none of the $y$ 's are 
in $\cap_{i=1}^{i=m} U_{i}$. Thus $x \notin U_{m}^{\prime}$ for $m$ sufficiently large.

Since $U_{i}^{\prime}$ does not restrict the first $n$ factors, $B$ is in $\mathrm{B}\left(\cup_{i>n} \beta_{i}\right)$ for any $n$. Thus $B$ is a tail event and hence zero-one, which means meager or residual.

COROLlARY. A BP measurable map from a countable product of spaces which is a Baire space to a second countable $T_{1}$ space is constant on a residual set if it does not depend on any finite number of factors.

Proof. In the circumstances described, the inverse image of a basic open set must be a tail $B P$ set, hence either residual or meager. Since the base is countable and since each point has an image, the Baire category theorem tells us that some basic open set must have residual preimage. Consider the set $S$ which is the intersection of the basic open sets with residual preimage. It must have residual preimage and hence cannot be empty. Suppose $x$ and $y$ are members. Because the range is $T_{1}$ the complement of $x$ and the complement of $y$ are open. One of the two must have residual preimage and thus contain a basic open set with residual preimage, which shows that the point whose complement had residual preimage is not in $S$. Thus there is at most one member; so $S$ is a singleton. The map is constant on the preimage of $S$.

This result extends the results in [6], [2], and [3] both by weakening the conditions on the product space and by requiring only $T_{1}$ and not $T_{2}$ in the range. The version in Andersen and Christensen [1] uses further conditions on the product to eliminate second countability on the range.

The Hewitt-Savage Zero-One Law is a bit more complicated than the Kolmogorov law and we will be able to prove it under somewhat more restrictive conditions on the independence theory, conditions capturing what is needed of the structure of a product measure without actually determining what a product independence theory is.

Definition. A family $\left(A_{i}\right)_{i \in \Gamma}$ is a test family for $A$ if whenever $B$ is independent of each of the $A_{i}, B$ is independent of $A$.

An independence theory is testable if every element has a test family and furthermore:

(1) if $\left(A_{i}\right)_{i \in \Gamma}$ and $\left(B_{i}\right)_{i \in \Gamma}$ are test families with the same index set and $\left(A_{i}\right.$, $\left.B_{i}\right) \in I$ for each $i$, then $(A, B) \in I$;

(2) if $\left(\gamma_{i}\right)_{i \in \Gamma}$ is a family of independence preserving morphisms fixing $A$ and $\left(A_{i}\right)_{i \in \Gamma}$ is a test family for $A$ then $\left(\gamma_{i} A_{i}\right)_{i \in \Gamma}$ is a test family for $A$.

EXAMPLES.

(8) A product measure space $\prod_{i \in N}\left(X_{i}, \mu_{i}\right)$ with all factors the same is testable using cylinder sets on a finite number of factors. The test sequences are the $A_{n} \in \prod_{i=1}^{i=n} X_{i}$ with $\mu\left(A_{n} \triangle A\right) \rightarrow 0$ as $n \rightarrow \infty$ used in the conventional proof of Hewitt-Savage.

(9) In a product of topological spaces $\Pi\left(X_{i}, T_{i}\right)$ the restricting factors theory is testable. The test families are obtained by considering finite unions of basic open sets contained in the set to be tested.

Theorem (Hewitt-Savage Zero-One Law). If $(X, I)$ is a testable indepen- 
dence theory and $G$ is a group of endomorphisms of $X$ which preserve independence and such that any $A \in X$ has a test family $\left(A_{i}\right)$ such that $A_{i}$ is independent of $\gamma_{i} A_{i}$ for some $\gamma_{i} \in G$, then any element $A$ fixed by all of the elements of $G$ is zero-one.

Proof. By (2) in the definition of a testable theory and the hypothesis, $\left(A_{i}\right)$ and $\left(\gamma_{i} A_{i}\right)$ are test families for $A$. But $A_{i}$ and $\gamma_{i} A_{i}$ are independent, so $A$ is zero-one.

Corollary (Extended Hewitt-Savage Law [4]). If $(X, \mu)$ is a recurring product measure, then any set fixed by any finite permutation of the indices is zero-one.

Proof. The hypotheses of the corollary state that the group $G$ of finite permutations interchanging factors with the same measure satisfies the hypotheses of the theorem. Since $G$ is a subgroup of the group $F$ of all finite permutations, invariance under $F$ will imply invariance under $G$. Applying the theorem tells us that the invariant events are zero-one.

Corollary (Category Analogue of the Hewitt-Savage Zero-One LAW [8]). In a countable power $\Pi(X, T)$ every BP set which is invariant under all finite permutations is either residual or meager.

PROOF. In the restricting factors theory for a topological product, the group of finite permutations of factors satisfies the hypotheses of the theorem when test families using finite unions of basic open sets are used. Hence any invariant open set is zero-one; that is, it is either all of the space or empty. For this part of the proof we do not need countability of the power.

A $B P$ set which is $G$ invariant will differ from an invariant open by the orbit of a set of first category. Under the hypotheses given, the group $G$ is countable, so the orbit of a set of first category is of first category. Thus an invariant $B P$ set must be meager or residual.

I see no way of modifying this proof to eliminate the countability condition on the power which distinguishes this corollary from the result of $M$. and $K$. P. S. Bhaskara Rao [8].

\section{REFERENCES}

1. N. J. M. Andersen and J. P. R. Christensen, Some results on Borel structures with applications to subseries convergence in abelian topological groups, Israel Math. J. 15 (1973), 414-420. MR 48 \#6308.

2. J. P. R. Christensen, Borel structures and a topological zero-one law, Math. Scand. 29 (1971), 245-255. MR 47 \#2021.

3. New York. MR 50 \# 1221.

4. S. Horn and S. Schach, An extension of the Hewitt-Savage zero-one law, Ann. Math. Statist. 41 (1970), 2130-2131. MR 42 \#3821.

5. M. Loève, Probability theory, Van Nostrand, Princeton, N. J., 1955. MR 16, 598.

6. J. C. Oxtoby, Cartesian products of Baire spaces, Fund. Math. 49 (1961), 157-166. MR 25 \#4055. 
7. Measure and category, Springer-Verlag, Berlin and New York, 1971.

8. K. S. P. Rao, S. Bhaskara Rao and M. Bhaskara Rao, A category analogue of the Hewitt Savage zero-one law, Proc. Amer. Math. Soc. 44 (1972), 497-499. MR 49 \#9823.

9. H. E. White, Two unrelated results involving Baire spaces, Proc. Amer. Math. Soc. 44 (1974), 463-466. MR 49 \#11486.

Department of Mathematics, Vassar College, Poughkeepsie, New York 12601

Current address: Department of Mathematics, Illinois Wesleyan University, Bloomington, Illinois 61701 